\title{
LA AMBIVALENCIA DE LOS VÍNCULOS EN EL CONSUMO: UN ANÁLISIS SOCIOLÓGICO DEL CASO HARLEY-DAVIDSON
}

\section{THE AMBIVALENCE OF BONDS IN CONSUMPTION: A SOCIOLOGICAL ANALYSIS OF THE HARLEY-DAVIDSON CASE}

\author{
Marc Barbeta Viñas \\ Departament Sociologia \\ Universitat Autònoma de Barcelona, España \\ marc.barbeta@gmail.com
}

\section{Cómo citar / citation}

Barbeta, Marc (2019) "La ambivalencia de los vínculos en el consumo: un análisis sociológico del caso Harley-Davidson". OBETS. Revista de Ciencias Sociales, 14(1): 17-48. doi: 10.14198/OBETS2019.14.1.01

\begin{abstract}
Resumen
El artículo propone un análisis del sentido de las relaciones desarrolladas en el consumo de Harley-Davidson. Se discute, a partir de evidencias empíricas, las tesis acerca de los vínculos en el consumo de marcas como nuevas formas de vínculo social. Se presentan los resultados de una investigación cualitativa realizada con grupos de discusión y entrevistas abiertas. Los resultados revelan que las relaciones en el consumo de Harley mantienen una estructura significativa relacionada con aspectos culturales, identitarios y emocionales, y mediados por las posiciones sociales de los consumidores. Asimismo, profundizan en los procesos de fragmentación y particularismo de la vida social, en el espacio del consumo.
\end{abstract}


Palabras clave: consumo, relaciones, marcas, comunidad, análisis discurso

\begin{abstract}
The article we propose the analysis of the meaning of the relations developed in Harley-Davidson consumption. From the empirical evidence, the theses about the bonds in brands consume as new forms of social bond are discussed. We present the results of a qualitative research carried out with focus groups and semi structured interviews. The results reveal that the relations in Harley's consumption maintain a diverse structure, related to cultural, identity and emotional aspects, as well as mediated by the social positions of the consumers. However, in addition to being part of a logic of community reconstruction and solidarity, they seem to deepen the processes of fragmentation and particularism of social life in the sphere of consumption.
\end{abstract}

Keywords: consumption, relations, brands, community, discourse analysis

\title{
Extended abstract
}

Several authors have analyzed the bonds and collective experiences that the consume of brands entails. The research focusing in 'brand communities' make this. A great extent of these proposals tends to conceptualize the bonds in consume as 'exits' to the individualizing process that distinguishes the development of modern times. As such responses oriented to the reconstruction of the bonds in community and social solidarity in contemporary society. These perspectives fundamentally analyze consume in the cultural dimension, as epicenter of such a process of reconstruction.

In the present work we suggest an alternative interpretation of the bonds that arise from consumer practice, as well as for the implications for the debate on sociability. We wish to defend the thesis that refers to the ambivalent nature of consumer bonds and highlighting the coexistence and simultaneous elements that may enter into contradiction in their development. We aim to unfold such insight and contribute to the debate introduced by the study of the Harley-Davidson brand, widely explored in literature.

Considering that the objective of this research is the analysis of the consumer bonds, we have considered the specific context of HarleyDavidson for its precedents in scholar research and the empirical evidence provided, or partially provided, in this group form of motocycle consume. It is a brand and a form of consume that furthermore complies with the criteria defined by Muñiz y O'Guinn for 
the formation of community bonds, and thus suggests an ideal context for the analysis; since this is a form of consume that is susceptible to be shared, as well as being a brand with strong image, and a rich and long history.

The analysis that we introduce is based in both method and qualitative empirical evidence. The questioning of the sense that bonds impose, for its correspondence with the proposal introduced in the research, in a qualitative orientation, and dedicated to the case of Harley-Davidson and the brand communities, that have fundamentally developed ethnographical methods of participative observation. In contrast, we hereby introduce the techniques of focus groups and semi-structured interviews. In any case, these techniques are usually prescribed as the most adequate in the attempt to capture both representations and social images of definite objects, as well as for the social reconstruction of the conducts of certain social processes. Specifically, focus groups allow us to analyze the union process and reintegration within the group, and observing in the construction of its discourse the element that relates to its participants. Interviews are the appropriate method for capturing the decision that the experience entails. Furthermore, the interest of focus groups and interviews are essentially determined macro-sociologically, and the subjects interviewed are considered as subjects that enjoy certain position in the structure inasmuch as they belong to certain social class, vital cycle or specific consumer group within Harley Davidson. This allow us to research on the hypothesis of non-hegemony of the consumer bonds, and to open our sample to consumers that belong to certain groups of Harley Davidson. The ethnographic approach usually restricts the analysis in a specific area or group and discard the rest.

This sample has been designed with the objective to capture the different images and experiences of consumer bonds that Harley Davidson consumers experience in accordance to their social position and relations with other groups of consumers of this brand. In this manner the socio-demographic variables of sex-gender, social and economic status and age are fundamental for the election of interviewees. On the other hand, the interviewed have to have purchased, at least, one Harley Davidson motorcycle, and aspect that provides flexibility in focus groups in which consumers of other 'custom' motorcycles are mixed. The variable corresponding to 'organic relation that the group maintains with Harley Davidson Inc.' has also been used for an heterogenic election of interviewees: informal interviews with staff of this brand have allowed to establish this specific variable. We likewise establish a typology of group that individual consumers feel more or less close with: 'HOG' (official group); 'Harley Space' (groups related to official store); 
'autonomous' groups (without organic relations with the company); 'Motoclubs' (without organic relations with the company). Perhaps a limitation that may be stressed in the construction of the sample has been the non-inclusion of all the diversity of groups, and thus it may possibly not cover the totality of the discourse's possibilities.

As such, we will follow the 'social-hermeneutic' model of sociological analysis introduced by Alonso Conde. Thus, firstly, it is carried out a semantic analysis of speech combined with a structural analysis. Secondly, a pragmatic analysis of discourse is performed where the narrated texts are related to their specific social contexts of production. In this paper we focus on the discursive dimension of the references to the 'others', which allows addressing, specifically, the semantic space (on the links consumption of Harley-Davidson (bonds, relationships or 'social bonds').

In the analyses of relations, we may observe at least three distinctive models that respond to three manners of experiencing and living the relations of consume. The institutionalized model that arises from the discourse of the middle social and economic positions, in which the group consume is affirmed as fundamental, although the relations may prove to be of a changing nature, weak and without evident implications to others; in which we find dynamics that express the recognition of others, as well as the necessity for limits that regulate relations while at the same time taking place identification narcissistic processes arising from the similarities between the 'harlist I' of consumers, in where the other is an extension of oneself or it is valued for the similarity with 'me'. The individualist model arises from the discourse in middle and middlehigh positions and rejects the relations of consume and where a particular strategy of distinction is carried out, only occasionally accepting? consume relation with friends and known people. The motoclub model, not exclusive of $\mathrm{HD}$, arises from the discourse in middle-low positions in where are fundamental strong relations and compromise. Some social and cultural aspects are associated to class position and projections of the 'ideal I' on the group and rider identity, being these key aspects of these bonds. However, it does not stop revealing a 'groupality' tending to be private and particular that works with internal codes, and thus losing individuality in favor of the group and thus against the autonomy of the individual.

These results, beyond making evident the Harley consume bonds and their interpersonal relation, references to others arising from usual situations, identities, values and common interests, or the more or less collective construction of the bond's sense, even in the cases of rejection, also show us how consumer bonds tend to fundamentally be oriented to distinction and group identity. The distinction with those perceived 
different, whether being for their practice of consume, their desires, codes, styles, values and used norms; a distinction that also expressed the symbolic struggle in and between social groups in the same segment/brand of consumers, and that makes evident a game to frequently distinguished by competition and exclusion for a symbolic power and social recognition. As we seen, the identification acquires a clear particular character, private and identity of a small group with a symbolic universe also particular and self-referred, whether being stronger or weaker bonds, and showing a major or minor degree of compromise with the others and the group. Therefore, we did not observe in the research any trace of clear 'social bond' in the manner we defined (see the paper). The consume of brands, and particularly HarleyDavidson, can hardly represent a context of mutual acceptance among consumers, based on the recognition of others as others, since it is inscribed in the so-called narcissism of small differences. Selfdefinitions and similarities, desires, symbols and coinciding codes together with the approval, narcissism and recreational recognition is practically the only motivation and reason to count on the other. It is well possible, that once dismantled the institutionalized mechanism of the Keynes-Ford and life in terms of mercantilism of an era where the private consume is shown as an area of reconstruction of identities and socialization that in the last years entered into crisis. We may grasp with this work the reinforcement of such this hypothesis and add that it is a failed "solution" of the reconstruction of solidarity and 'social bonds', inasmuch in consume it is lacking a project of global universal integration for the reconstruction of our societies.

\section{Introducción}

El análisis de los vínculos desarrollados en el consumo de marcas se ha presentado como un objeto de interés creciente para la investigación social. La literatura reciente viene analizando a través de categorías como "comunidad", "tribu" o "subcultura", los vínculos desarrollados entre consumidores de marcas determinadas, así como las experiencias colectivas derivadas de sus prácticas de consumo. Estos vínculos comunitarios, no siempre de forma explícita, han sido interpretados como ejemplos de reconstrucción de la solidaridad y de los vínculos sociales. En definitiva, como salidas al proceso de individualización que habría caracterizado el desarrollo de la modernidad tardía, siendo el ámbito del consumo uno de los epicentros de dicha reconstrucción. 
Con el presente trabajo se pretende contribuir a este debate atendiendo a la dimensión relacional de los vínculos entre consumidores de la marca Harley-Davidson (H.D.) desde una perspectiva sociológica. Se persigue ampliar, así como en algunos aspectos confrontar, parte de los hallazgos de las investigaciones centradas en el consumo de HD circunscritas en el ámbito anglosajón. Para ello partimos de la hipótesis de trabajo según la cual los vínculos en el consumo de marcas se expresan en términos de ambivalencia; es decir, mediante la coexistencia y simultaneidad de elementos que entran en contradicción entre sí en su génesis y desarrollo significativo (Laplanche y Pontalis, 1967; Merton y Barber, 1976).

Con este planteamiento atenderemos a los usos sociales de la marca HD en tanto que remiten a determinadas formas de sociabilidad, estrategias y experiencias de consumo contradictorias entre sí, insuficientemente estudiadas. Proponemos analizar así el sentido colectivo de los vínculos desarrollados en los contextos de consumo de HD, así como su configuración motivacional. Con el fin de abordar estos aspectos, se analizará la dimensión sustantiva de los vínculos desarrollados en el consumo de HD. Nos preguntamos en calidad de qué los 'otros' son tenidos en consideración (más o menos conscientemente) en la formación de dichos vínculos. En segundo lugar, nos interrogamos en qué medida estos vínculos en el consumo generan o expresan lo que definiremos como 'vínculo social'. El trabajo plantea, de este modo, investigar cómo los consumidores desde contextos sociales específicos significan intersubjetivamente sus vínculos y experiencias en el consumo. Asimismo, pretende cubrir el vacío existente derivado del escaso número de investigaciones sobre vínculos en el consumo de marcas usando grupos de discusión y entrevistas abiertas. Contribuimos así a la literatura sobre marcas en general y HD en particular, analizando, en continuidad con otros trabajos (Barbeta Viñas, 2018), las relaciones generadas en las prácticas de consumo atendiendo a las representaciones e imágenes de estas.

\section{El análisis de los vínculos en el consumo: entre la individualización y la reconstrucción comunitaria}

En las últimas décadas determinadas prácticas de consumo de marcas han sido asociadas a modos específicos de vínculo por un amplio número de trabajos. Estas formas de consumo en las que el contacto con los 
demás deviene un aspecto central, no obstante, se han valorado como ejemplos de la debilidad de dichos vínculos. Así han sido enmarcadas en los procesos de individualización y privatización de la vida social que caracterizan la modernidad tardía (Lasch, 1979; Sennett1979 y 2012; Bauman, 2001b). Con un acercamiento más bien teórico, autores como Bauman (200la y 2007) y Lipovetsky (1983 y 2006) han analizado estas formas de consumo como 'narcisismo colectivo', expresión del 'hiperindividualismo' dominante en la actualidad, en el que los vínculos en el consumo, cuando se producen, mantendrían un carácter frágil, volátil y fugaz.

En contraste, otros trabajos que parten del mismo diagnóstico sobre el avance del individualismo en la modernidad han interpretado estos vínculos en el consumo como reacción al mismo. Estas formas grupales de consumo serian entonces parte de la reconstrucción de los vínculos sociales y de la solidaridad (Petterson y O'malley, 2006). Entre estos trabajos encontramos quienes señalan el carácter inestable y cambiante de los vínculos en tanto que expresión del modelo posmoderno de socialidad (Maffessoli, 1988; 1996; Blocker Houston y Flint, 2012). Otros autores, en cambio, analizan la solidez y la fortaleza de estos vínculos del consumo. Entre estos últimos trabajos se ha destacado el carácter subcultural y comunitario de algunos de los vínculos en el consumo de marcas (Cova et.al, 2007; Cova y Cova, 2002; Muñiz y O'Guinn, 2001; Schouten y McAlexander, 1995). Incluso se ha señalado el carácter prioritario y central del vínculo respecto al objeto/marca en el consumo (Santos et.al, 2017; Marzocchi et.al 2013; Cova, 1997).

Estos estudios, la mayor parte de ellos inscritos dentro de la Consumer Culture Theory (Arnould y Thompson, 2005) y elaborados con base empírica de apoyo, han abordado los aspectos que contribuyen a configurar y mantener los vínculos alrededor del consumo de una marca. En este sentido, Muñiz y O'Guinn (2001), inspirados en los clásicos Tönnies y Durkheim, establecen tres aspectos básicos que definen la sociabilidad comunitaria de los consumidores de ciertas marcas, fundamentalmente, en base a componentes homogéneos. Los resultados de otros trabajos dirigidos al análisis de marcas distintas presentan resultados coincidentes. Entre ellos, destacamos los trabajos dedicados a Harley-Davidson.

1) La conciencia de pertenencia: permite a los miembros de la comunidad definirse identitariamente: aquello que son y a lo que se oponen (Muñiz y O'Guinn, 2001; Schouten y MacAlexander, 1995; Carlson, Suter y Brown, 2008; Bagozzi, y Dholakia,.(2006) también expresa una unión 
en sentido más emocional que solamente instrumental (Cova 1997; Martin, Schouten, McAlexander, 2006). 2) Los rituales y tradiciones compartidas: se comunican significados, valores y normas propias de la comunidad; también se configura la historia del grupo, el relato acerca de la marca, así como experiencias comunes, siendo una fuente de significados para la marca y el grupo (McAlexander, Schouten y Koenig, 2002; Schembri, 2009; Schouten y McAlexander, 1995; Belk y Tumbat, 2005). 3) La responsabilidad, el compromiso y la implicación con la comunidad y sus miembros: existe el deber de integración de los nuevos miembros, de defender la imagen de marca ante los no miembros, y regenerar sus significados. La integración satisfactoria aumenta la lealtad con la marca y los demás consumidores (Cova, Kozinets y Shanckar 2007; McAlexander, Kim y Roberts, 2003; Aaker, 1991).

Por otra parte, se ha dedicado atención a los aspectos más conflictivos de los vínculos en el consumo. Pero, como hemos afirmado, se trata de una perspectiva todavía insuficientemente desarrollada. Los trabajos de Ewing et.al (2013), Muñiz y Hamer (2001), McAlexander, Schouten y Koenig (2002), McAlexander (2011), Hickman y Ward (2007) o Fournier et.al (2000), señalan aspectos parciales como las barreras de salida del grupo y el abandono de las comunidades, las jerarquías y clasificaciones simbólicas entre consumidores 'rivales' o dentro de la misma comunidad, las conductas 'delictivas', etc. Otros trabajos han señalado la emergencia de conflictos en las comunidades derivados de la competencia y oposición de intereses entre sus miembros (Gebauer et.al, 2012). Conflictos que, en todo caso, sean mostrado ambivalentes para el desarrollo de las mismas (Husemann, et.al, 2015).

\section{Aproximaciones teóricas alternativas para el análisis del sentido de los vínculos en el consumo}

Los trabajos anteriores generalmente han tendido a descuidar los puntos siguientes, básicos para una mejor comprensión de los vínculos en el consumo: a) la dimensión contradictoria de los vínculos en el consumo; b) una concepción de los vínculos configurados únicamente por la proximidad estética e identitaria de los consumidores. Esta visión no haría más que reactualizar la solidaridad mecánica durkheimiana, con las siguientes implicaciones: primero, no se problematizan unos vínculos que se presentan en su versión más aislada, fragmentada y mico-grupal en diversos contextos de consumo, descuidando el nivel macrosocial de 
este proceso (Alonso y Fernández Rodríguez, 2013). Segundo, se toma el consumo únicamente en su dimensión estético-cultural, omitiendo que las prácticas de consumo están también ligadas a las posiciones sociales y a formas de expresión afectiva de los consumidores (Lee, 1993).

Parte de la sociología del consumo, en cambio, ha situado las posiciones sociales, definidas en términos de clase social y/o estatus, como matriz de los vínculos en el consumo. Es el caso de los trabajos clásicos de Veblen (1899), Simmel (1911), Weber (1922) y Bourdieu (1979), donde se analiza cómo el consumo deviene una vía para la configuración de grupos, mediante lógicas de imitación y diferenciación respecto los grupos de referencia y/o pertinencia, propios y ajenos. Aquí los vínculos en el consumo no se interpretan, en lo fundamental, como ningún tipo de reconstrucción comunitaria, sino más bien como parte de las luchas simbólicas existentes entre grupos sociales.

De acuerdo con estos trabajos, no podemos desdeñar el condicionamiento que ejerce la estructura social sobre el consumo, tal y como se constata que ocurre en la realidad social estudiada (Borràs, 1998). Para ello, hemos de tener en cuenta que las posiciones sociales tienden a proyectarse en los estilos de vida e identidades desarrollados en los vínculos del consumo. Prácticas enclasables, manifestación de los gustos, así como la apropiación del significado y uso de objetos y marcas, constituyen espacios donde se expresa y suele cristalizar dicho condicionamiento (Bourdieu, 1979; Miller 1987; Chaney, 1996; Grignon y Passeron, 1992). Los estilos de vida y las identidades con las que se dinamiza el consumo están también vinculadas con las representaciones que los consumidores, desde posiciones sociales específicas, realizan de los otros consumidores y sus prácticas. Lo que implica algún tipo de vínculo por referencia a los otros.

Dentro de la antropología se ha destacado la práctica del consumo como un quehacer colectivo. Los consumidores, más allá de semantizar a objetos y marcas, se orientarían por las prácticas de dar y recibir, de relación con los demás, de gustar y mostrar afecto u odio, así como construir relatos culturales e identitarios (Mauss 1924; Miller, 1998; Douglas y Isherbood, 1979; McCracken, 1988; Sherry, 1983). Los trabajos acerca de las comunidades marca se han centrado en alguno de estos aspectos en particular (véase Fournier, 1998; con carácter general: Petterson y O'malley, 2006).

Finalmente, algunos modelos teóricos nos acercan a ciertos aspectos de la dimensión afectiva y psicosocial de los vínculos en el consumo. En 
este sentido pueden ser útiles conceptos desarrollados originalmente por Freud $(1914 ; 1921)$ y posteriormente por otros autores como los que dan cuenta de la formación grupal y los modelos de vínculo que las personas mantenemos con los demás (Wallace y Baumeister, 2002; Buss y Chiodo, 1991; Bowlby 1979). Desde esta perspectiva distintos trabajos muestran que el objeto (líder, idea) que permite la identificación entre consumidores, así como su orientación conductual sería en estos momentos los deseos, los estilos de vida y los valores simbólicos relacionados con determinadas marcas y objetos de consumo. Se destaca también que los vínculos en los procesos de consumo actuales estarían fuertemente marcados por su carácter narcisista, fragmentario, de alta competitividad, y promovidos fundamentalmente por la satisfacción de los deseos y aspiraciones de los consumidores (Ciseck et.al, 2014; Sedikides et.al, 2007; Severiano, 2005; Minsky, 1998).

\section{Los modelos de vínculo en el consumo: lazos, relaciones y 'vínculos so- ciales'}

La literatura sobre las formas de sociabilidad en el consumo de marcas ha atendido a los vínculos en alguna de sus diferentes formas: vínculos cara a cara, psicológicos e incluso redes de conexión virtual. No obstante, no se ha planteado una delimitación teórica sistemática acerca de las distintas formas de sociabilidad en el consumo. A continuación, se presentan algunas categorías que tienen como fin elaborar un marco analítico útil para la diferenciación de los diversos tipos de vínculos en las prácticas de consumo y facilitar así el análisis de su sentido (Barbeta Viñas, 2014).

Proponemos diferenciar tres formas de vínculo, siguiendo los planteamientos de la sociología clásica: lazos, relaciones y 'vínculo social'. Cabe señalar, no obstante, que lo que aquí abordamos son formas de sociabilidad humana y no vínculos de personas con cosas en tanto que relaciones sociales objetivadas (Marx, 1844).

Conceptualizamos las relaciones, como una conducta plural -de varios- que, por el sentido que encierran, se presentan como recíprocamente referidas, orientándose por esta reciprocidad (Weber, 1922). En las relaciones el contacto no es unilateral; y además, no pueden confundirse con la interacción: mientras esta responde a la dimensión objetiva del contacto con los demás, la relación encierra un significado intersubjetivo entre sus participantes (Habermas, 1980). 
Los lazos responden a la presencia unilateral del otro, pasado, presente o imaginado, en la orientación de las actitudes y conductas, según las perspectivas de Weber (1922) y Freud (1921). Sin embargo, el análisis de los lazos queda fuera de los objetivos de este trabajo, habiéndose desarrollado en Barbeta Viñas (2018).

En cuanto al 'vínculo social' lo conceptualizamos como una dimensión atributiva y variable de lazos y relaciones. Estas responderían a formas particulares de vínculo, siendo la dimensión propiamente 'social' una cualidad que tienen algunos de los vínculos. Esta dimensión social se establecería por el sustrato emocional del amor proyectado en el contacto con los demás como aceptación mutua (Maturana, 1988). Por lo tanto, no toda forma de vínculo sería social, pues existen otras dimensiones como los intereses, el cálculo, etc., que no son fundamentos de lo social, aunque todas ellas se solapen en los contactos con los demás. El 'vínculo social' responde a la aceptación del otro en tanto que otro, en su plena alteridad. Es el tipo de vínculo en que el otro nos hace falta como otro, no lo que podemos conseguir de él, o tomarlo como extensión de uno mismo (Boltanski 1990; Izquierdo, 2003). Este reconocimiento no sólo cabe pensarlo en términos de individuos, sino por la pertenencia a grupos con determinados estilos de vida e identidades como las que se dan en las prácticas de consumo. En el 'vínculo social' se trata de atender a la experiencia de realización subjetiva del otro, y atender a lo que el otro experimenta de nuestro vínculo con él, quedando así excluida de lo propiamente social la dimensión objetivadora de los vínculos (Izquierdo, 1998).

\section{Metodología, diseño y contexto del estudio}

El análisis que presentamos se basa en una metodología y unas evidencias empíricas de tipo cualitativo. Hemos escogido el contexto específico que representa el consumo de Harley-Davidson por las evidencias existentes en la literatura de constituir un modelo de consumo que en muchas ocasiones se presenta con un carácter grupal. HD cumple con los criterios que señalan Muñiz y O’Guinn (2001) para la formación de vínculos comunitarios, lo que sugiere un contexto idóneo para el análisis propuesto. Se trata, además, de una marca con una imagen fuerte, con una historia larga y rica en términos simbólicos (Yates, 2000: Schouten y McAlexander, 1995). 
Los trabajos de orientación cualitativa dedicados al estudio de H.D. y a las comunidades marca en general han desarrollado, fundamentalmente, métodos etnográficos. En contraste, nuestra aportación metodológica propone el uso de técnicas como grupos de discusión (GD), grupos triangulares (GT) y entrevistas abiertas (EA), siguiendo la tradición sociológica de Alonso (1998) y Conde (2008). Estas técnicas suelen prescribirse como las más adecuadas en el trabajo de captar las representaciones e imágenes sobre objetos determinados, así como para la reconstrucción de las actitudes ante procesos como los vínculos en el consumo.

Los grupos de discusión nos permiten analizar el proceso de unión del grupo, observando en la construcción discursiva del mismo, aquellos sentidos del consumo mediante los cuales se vinculan sus participantes. La entrevista abierta resulta adecuada para captar el decir de la experiencia. Dado que el interés de los grupos de discusión y las entrevistas abiertas es macro-sociológico, los sujetos entrevistados son considerados como sujetos que ocupan una posición estructural en tanto que pertenecen a determinadas posiciones socio-económicas, ciclos vitales o grupos concretos de consumidores de HD, como detallamos en la siguiente figura. Esto nos permite trabajar con la hipótesis de no homogeneidad absoluta de los vínculos entre consumidores, y abrir la muestra a consumidores que forman parte de grupos distintos dentro del segmento Harley. La aproximación etnográfica suele restringir el análisis en un ámbito/grupo específico, dejando de lado el resto. Cabe añadir que las técnicas aquí utilizadas tienden a favorecer un análisis de los discursos más legítimos y codificados de los grupos sociales, si bien los GT y las EA posibilitan abrir los códigos del discurso y captar los elementos experienciales. Entre sus limitaciones está, por tanto, dejar de lado el nivel de los hechos y el análisis de los comportamientos.

El diseño de la muestra en su conjunto y la composición de los grupos y entrevistas abiertas en particular pretenden obtener información sobre el sentido que dan a los vínculos y experiencias en el consumo de Harley consumidores que ocupan diferentes posiciones sociales. No pretendemos investigar ningún agregado de discursos individuales, ni un universo poblacional. El muestreo realizado es de carácter estructural y teórico; busca representar un universo discursivo vinculado con macro-grupos sociales y estilos de vida pertenecientes a la realidad social objeto de estudio. El trabajo de campo forma parte de un proyecto más amplio desarrollado entre 2011 y 2013, sin relación con ninguna investigación comercial. Así algunos de los objetivos que se perseguían estaban bien 
apegados al análisis de los vínculos que aquí se plantea. La contactación, elaboración de entrevistas y grupos, así como la transcripción del material y análisis ha sido llevado a cabo por el responsable de la investigación.

El contexto de la investigación (Askegaard y Jeppe, 2011) queda circunscrito a las siguientes variables socio-demográficas mediante las cuales se han seleccionado los entrevistados buscando cierto equilibrio entre homogeneidad y heterogeneidad en la composición de los grupos de discusión (figura 1). Por otra parte, todos los entrevistados seleccionados tienen que haber adquirido -por lo menos- una motocicleta Harley-Davidson (cuestión que se flexibiliza en un grupo de discusión en el que se han mezclado consumidores de motocicletas 'custom' de distintas marcas).

Figura 1. Esquema del diseño técnico de la investigación.

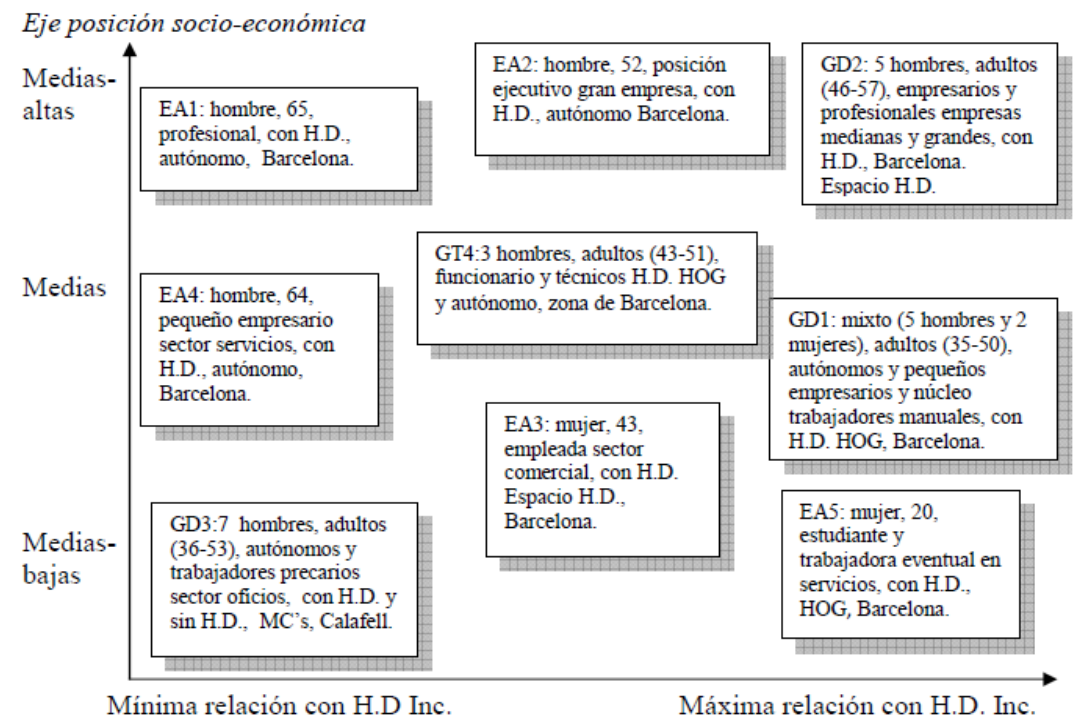

Eje tipos de grupo de consumidores por relación orgánica con la empresa/marca

Fuente: Elaboración propia.

Finalmente, la variable 'relación orgánica que el grupo mantiene con la empresa H.D. Inc', ha sido utilizada para dar heterogeneidad entre los grupos y en el conjunto de las entrevistas. Hemos distinguido consumidores: 'HOG' (grupo oficial Harley Owners Group); 'Espacio Harley' 
(grupo vinculado a establecimiento comercial en Barcelona); grupos 'autónomos' (sin vinculación orgánica con la empresa); 'Motoclubs' (sin vinculación orgánica con la empresa pero autodefinidos como tales). Una limitación que debe reconocerse en la muestra es la no inclusión de toda la diversidad de tipos de grupo, especialmente dentro de las diferencias que muestran los MC's entre ellos. De este modo, puede no verse cubierta la totalidad de la variabilidad discursiva.

Seguimos el modelo sociohermenéutico de análisis sociológico del discurso planteado en Alonso (1998) y Conde (2009). Se propone, así, un análisis semántico, combinado con un análisis pragmático del discurso sobre los vínculos, donde éste se relaciona con sus contextos sociales de producción. La micro-situación de los grupos de discusión y entrevistas abiertas nos sirve como analizador de la macro-situación social, circunscrita en este caso al segmento de moteros Harley. La labor interpretativa, en parte, surge de entender los discursos sobre los vínculos como reflejo de los contextos sociales de referencia. En este trabajo nos centramos en el espacio semántico relativo a las relaciones (sean o no expresión de 'vínculos sociales') del consumo de Harley-Davidson.

\section{Resultados y discusión: Relaciones entre consumidores de Harley- Davidson}

Presentamos los aspectos más relevantes del análisis de las relaciones. En consistencia con la literatura revisada, las relaciones en el consumo de HD adquieren en los discursos un papel central. Su análisis revela tres modelos distintos de relaciones, variables según las posiciones sociales de les consumidores, así como según el tipo de grupo con el que se consume. Cada modelo representa un "tipo ideal" weberiano las referencias empíricas de los cuales tienden a predominar en unos grupos de discusión y entrevistas abiertas o en otros.

\section{Comunitarismo (narcisista) lúdico: relaciones en el HOG}

Un primer discurso es el producido por los consumidores de posición socioeconómica media (con fracción trabajadora), mayoritariamente miembros del HOG (GD1, GT4, EA3).

Este discurso revela que las relaciones entre consumidores son una pieza clave en la elaboración del sentido de las prácticas de consumo de 
Harley. Como han señalado distintos trabajos (Schouten y McAlexander, 1995; McAlexander, Schouten y Koenig, 2002; Shcembri, 2009), estas relaciones son una vía fundamental para la configuración de la dimensión grupal del consumo. En base a éstas los consumidores adquieren una identidad colectiva y compartida; las experiencias de consumo, el ritualismo y el carácter ceremonial de sus prácticas (salidas, desfiles, etc.), contribuyen asimismo a mantener y actualizar tanto la identidad común, como los significados simbólicos de la marca y de su consumo. El discurso explícito incluso llega a usar términos como "familia" y "hermandad" para caracterizar estas relaciones de consumo. Ello es congruente con los resultados de otros trabajos acerca el carácter comunitario de las relaciones en las comunidades marca (Muñiz y O'Guinn, 1996; 2001; Cova et.al, 2007).

Atendiendo al nivel pragmático del discurso, los 'otros' del vínculo adquieren, en general, un papel auxiliar. La finalidad declarada de los vínculos en el consumo es fundamentalmente lúdico-festiva ("pasarlo bien", "la diversión"); y en base a ésta se proyecta sobre los demás una expectativa de contribución al divertimento en el consumo. Ello coincide con la tipología elaborada por Holt (1994) cuando recoge que lo lúdico del consumo tiende a producirse en el marco de relaciones interpersonales. Sin embargo, en el presente contexto, los discursos se refieren a la importancia del "buen funcionar" de los demás para el mantenimiento de las relaciones. Así, los 'otros' del consumo devienen medios para la diversión. Aquellos que por las razones que sean no "funcionan" -usando una expresión marcadamente instrumental-, aquellos con quienes no es posible pasarlo bien, no forman parte del universo de los posibles. La propia "autorealización" es la posición desde la que los consumidores se vinculan con los demás. Paradójicamente, el valor del "compañerismo" es característico de este discurso. Sin embargo, en la dinámica del GD, puede jugar un papel favorable a su legitimación y cohesión grupal.

Este papel auxiliar de los consumidores con quienes se consume más o menos habitualmente es coherente con el tipo de relaciones abiertas y frágiles que revela el discurso y que Bauman (2001a) señala como típicas de los procesos de consumo posmodernos. En este discurso no se relatan compromisos fuertes con los otros consumidores (salvo alguna excepción que deriva en una amistad que transciende las relaciones de consumo y es referida a un nivel estrictamente individual). Más bien existen narraciones donde se enfatiza en el desconocimiento personal, así como en el manifiesto desinterés por la vida de los demás miembros del grupo. 
En la medida en que la satisfacción personal y la diversión parecen ser los móviles de las relaciones, de lo que se trata es de dar con consumidores con quiénes conseguirlo. Cuando las relaciones no se adecuan a tales fines, se contempla la salida del grupo, sin que ello implique mayores consecuencias.

A diferencia de lo que plantea Cova (1997), este discurso revela que el compromiso se muestra con la marca y la identidad que proporciona, no tanto con aquellos 'otros' con los que un consumidor se relaciona en sus prácticas de consumo. Lo anterior es concordante con algunos trabajos que señalan que los vínculos en las comunidades marca no son los más significativos y relevantes de los consumidores, lo que no significa que no lo sean en alguna medida (Bardhi et.al, 2012; Muñiz y O'Guinn, 2001). A diferencia de lo que ocurre en otros modelos de relación, este discurso no expresa la existencia de restricciones importantes para la entrada y/o salida del grupo (siempre y cuando se consuma HD). Las relaciones adquieren, de este modo, un sentido dinámico y cambiante. Los discursos revelan el predominio de una óptica cuantitativa, no cualitativa de las relaciones: se valora la "suma" de relaciones (GT4). La cantidad de relaciones, y no sus características, es un valor propio y diferencial del presente discurso.

H (Hombre): "Sólo te puedo decir que a lo mejor hay gente que los conozco hace 15 años y no sé a qué se dedican. Ni me interesa. Me interesa que cuando vienen conmigo en moto nos lo pasamos bien, que sean buenos compañeros de ruta, funcionamos bien, buen rollo entre las mujeres también" (GD1). ${ }^{1}$

$\mathrm{H}$ : “...el objetivo es que tú has de encontrarte a gusto, y si tú te encuentras a gusto, pues continuas, reproduces aquella relación. De modo que, si vamos con un grupo, es porque nos encontramos a gusto. Y el objetivo es encontrarte a gusto nosotros mismos, con el grupo y con nosotros mismos. Si es así, perfecto. Que no, la misma dinámica lo va modificando. Nadie espera que de aquí salga ... yo que sé, una relación idílica, si no, tienes que encontrarte a gusto, que me imagino que es la pretensión que tenemos cuando vamos con la moto, y punto" (GT4).

Por otra parte, las normas internas del grupo, es decir, aquello que regula las relaciones entre sus miembros tiene como base el principio latente de justicia según el cual: todos los consumidores de Harley son iguales ante los demás. Así se expresa cierta consciencia entre los harlistas del

1 Algunos verbatims de grupos y entrevistas han sido traducidos al castellano. 
HOG que en sus relaciones con los 'otros' (cuando se circula, se convive, etc.) son necesarios determinados límites y restricciones a aplicar a todos por igual. Se trata de un principio de justicia que, elaborado teóricamente por Freud y otros autores (Forrester, 1997), así como vinculado con el sentimiento de envidia, funciona como elemento cohesionador de las relaciones intergrupales. En el caso analizado interpretamos que no se trata tanto de un acuerdo vinculante para todo el grupo, sino de experimentar que los 'otros' algo pierden o pueden perder, de no ceñirse a las mismas constricciones. Tal como se relata, cuando es un solo individuo quien pretende traspasar los límites comunes se cuenta con la posibilidad de expulsión del grupo (H:“...también hay gente que viene al grupo o estaba en el grupo, por ejemplo, y se quiere aprovechar también, eh, de la situación. A estos se los peina bien peinaos y no vienen más", GD1). De aquí se deduce que el riesgo de descohesión grupal en el HOG depende del número de consumidores con pretensión de privilegios. A diferencia de la dimensión anterior donde los 'otros' “sirven” para pasarlo bien, aquí el 'otro' se reconoce como sujeto de derecho, siendo ello condición para el desarrollo de las relaciones: "cuando vas en grupo te tienes que amoldar a lo que hay..." (GDl).

La identidad harlista, según observamos, juega un papel homogeneizador que cohesiona y excluye al mismo tiempo: se minimizan las diferencias internas entre consumidores a la vez que se enfatiza en las diferencias con los otros grupos, ya sean del segmento Harley o de otras marcas. Por ejemplo, estos consumidores reconocen "vetar", racionalizándolo y justificándolo, la entrada de aquellos motoristas que no consumen Harley. Ello sugiere que las relaciones entre consumidores se establecen fundamentalmente a partir de sus semejanzas. Semejanzas que suelen materializarse en características compartidas relacionadas con el uso de símbolos, signos y experiencias de consumo, valoradas e idealizadas como parte de la identidad harlista. Estos elementos del consumo, además, son apropiados por estos consumidores del HOG en el marco de un estilo de vida "familiar", "responsable", e integrados de forma no conflictiva con el resto de ámbitos de vida de los mismos consumidores. El ser padres o madres de familia, con trabajo y responsabilidades debe poder conjugarse sin problemas con la identidad harlista. Ello es consistente con distintos trabajos que, desde ópticas diferentes al presente trabajo, han señalado el papel de estos elementos como bases sobre las que se construyen las relaciones en el consumo (Santos et.al, 2017; Cova et.al, 2007; Schouten et.al, 2007). 
Ahondando en esta perspectiva, observamos como el reconocimiento y aceptación de los harlistas encuentra también su base en el modelo de la identidad personal harlista. El proceso de configuración grupal y de identidad colectiva se realiza a partir de aquello que sienten que son o quieren ser los consumidores, así como las similitudes en la identidad harlista que muestran entre sí los consumidores. Ello nos lleva a conjeturar, siguiendo otras investigaciones, un marcado carácter narcisista en estos procesos relacionales. El sentido de la relación se construye de acuerdo con el modelo de una identidad Yoica idealizada, basada en los atributos simbólicos típicos que se proyectan sobre los harlistas: rebeldía, libertad, rudeza, entre otros (Minsky, 1998; Ciseck, et.al, 2014; Sedikides et.al, 2007).

En definitivita, este discurso revela cómo del 'otro' tiende a buscarse la admiración propia, así como la confirmación de las 'bondades' del Yo harlista. Los 'otros' en esta comunidad de semejantes se conciben -por lo menos en parte-, como la extensión de uno mismo. El relato de los consumidores no solamente revela que ciertas diferencias entre motoristas pueden ser motivo de conflicto y exclusión. Muestra también la relevancia que adquiere en estas relaciones el sentimiento de familiaridad; es decir, aquello ya conocido en tanto derivado de uno mismo, aquello que nos recuerda algún elemento característico de nuestro Yo. En base a la identidad Harley, expresan en el GT4, "parecía que nos conociéramos de hace muchos años. Y realmente nos acabamos de ver". Así, el discurso se orienta por una autoreferencialidad que expresaría cómo la génesis de estas relaciones pasa por el encuentro de la propia identidad Harley en el 'otro'.

H: "No nos mezclamos, no nos mezclamos...

H: No, no es mezclar...

E (Entrevistador): Pero no iríais juntos...

$\mathrm{H}$ : No, pero no porque tenga nada contra las otras marcas, sino porque ya es algo de cajón.

M (Mujer): De sentido común, no. (...)

E: Con los Harleys o con otros...

H: Hombre, con los Harleys porque son los que más... relación hay.

$\mathrm{H}$ : Son los del mismo grupo "(...)

M: "No, pero es que la marca ya...

H: ...por decir algo, y tú llegas allí y tú vas vestido con tu chaleco, tus colores, con botas, Harley y tal, y te encuentras un tío que viene de Escocia, bueno estos porque con faldas de colores, pero te encuentras tíos de Alemania y tal, y si no fuera porque pone que son del club de Alemania tú 
dirías que pueden ser de aquí, porque vamos todos iguales, todos pensamos lo mismo, todos llevamos más o menos lo mismo rollo"(GD1).

\section{Individualismo y pequeño grupo particularista}

Otro discurso lo encontramos en los consumidores de posición socioeconómica media-alta del GD2, la EA2 y un núcleo del GT4 de posición media; suele predominar entre los consumidores entrevistados más veteranos.

En este discurso se configura una concepción individualista y solipsista del consumo de Harley. Este se entiende como una práctica no relacional, no tanto en el sentido de que nunca se consume con otros harlistas (dado que estos motoristas no siempre consumen solos), sino por la expresión del deseo o preferencia del consumo en solitario, así como por la visión de lo que significa ir en HD. Para estos consumidores lo más relevante en el consumo de Harley es la relación con su motocicleta, no con los demás. La experiencia de conducir HD, según se expresa en el discurso, es algo que se vive como una sensación "muy personal" (GD2) en la que los demás parecen quedar ignorados u obviados. Es justamente esta posibilidad (imaginaria) de prescindir de los 'otros', incluso en plena carretera, lo que posibilita un consumo ocasional en grupo. El consumo en pareja o con un pequeño grupo muy exclusivo ("con amigos escogidos", GD2) es el único modo no solitario de consumir que se contempla.

Cuando las relaciones aparecen en el discurso más allá de lo señalado lo hacen, fundamentalmente, para quedar desacreditadas. Se juzga como una falsa "exageración" (GD2) la caracterización "familiar" y comunitaria de las relaciones del HOG. De hecho, el rechazo a los 'otros' emerge de forma definitiva en las dinámicas cuando se caracteriza a una parte de los consumidores de HD como unos "cretinos totales" (GD2); también cuando se enfatiza que el consumo en grandes grupos va en detrimento de la diversión, dado que los demás aparecen como obstáculos para la misma. Tener que lidiar con las particularidades de cada uno, las idiosincrasias personales, por mucho que sean consumidores de Harley, es algo que se rechaza por completo. Incluso las referencias discursivas a los demás suelen aparecer en abstracto, no se encuentran en el discurso muchas referencias a otros concretos y reales, lo que evidencia la distancia que se mantiene con los demás y la ausencia efectiva de un modo comunitario y relacional de consumir Harley. Otros trabajos ya señalan la 
manifestación de oposición e incluso de cierta hostilidad hacia consumidores de marcas definidas como "rivales" (Ewing et.al, 2013; Hickman y Ward, 2007; Muñiz y Hamer, 2001). Sin embargo, aquí nos encontramos con procesos de rechazo explícito al 'otro' dentro del segmento de HD.

En el discurso sobre el consumo en pequeño grupo emergen también elementos que revelan el condicionamiento que las posiciones sociales de los consumidores ejercen sobre el mismo. Aparecen referencias a sus posiciones sociolaborales, así como a las de los miembros de sus respectivos pequeños grupos: profesionales liberales, directores de empresas multinacionales, etc. Referencias que, además, se acompañan de proyecciones de sus habitus comunes, expresivos de prácticas y gustos con capacidad distintiva. Desde de rutas exclusivas con HD por los Alpes franceses y por USA, hasta referencias con significaciones más populares como el baile country. Ello es revelador de una cierta amplitud en el gusto, así como de una capacidad de combinación de prácticas culturales y de consumo propia de las posiciones sociales y económicas medias-altas (Ollivier et.al, 2009).

H: "Sí, pero lo que pasa es que yo, por ejemplo, yo con mi mujer y todo esto siempre viajamos solos, siempre. O sea, tengo mi moto y era una relación yo con la marca, pero era mi moto. Entonces yo... que veía a otros harlistas, yo los saludaba, pero... todo lo que... todo lo que estaba alrededor... yo creo que la esencia estaba dentro; es simplemente la relación que uno tiene con su moto. En este caso, yo creo que Harley tiene una cosa especial que no lo puedo identificar pero es como una alma que tiene. Esa moto como te sientes tú con la moto!"

H:" (...), yo cada vez me he dado más cuenta que a mí me gusta ir solo!

H: A mí me gusta también, pero incluso hiendo en grupo hay esa posibilidad de que esto es una cosa tuya y que está dentro de ti. Yo cuando llevo grupos, por ejemplo, para ir a EUA, me he dado cuenta de que claro al fin y al cabo, no tengo que tratar con ellos como personas, posiblemente a una persona que es un cretino total en el grupo. Pero tienes que tratar con ellos nada más en el momento de comer. Cuando estás encima la moto, tampoco..." (GD2).

El conjunto de estos elementos nos lleva a conceptualizar la génesis de estas relaciones como una estrategia de diferenciación particularista (Bourdieu, 1980; Chaney, 1996). Adquirirían parte importante de su sentido en el proceso de diferenciación que estos consumidores llevan a cabo dentro del mismo segmento de la marca HD. Por tanto, su campo de 
operaciones contempla no solamente la estructura social y el sistema de marcas de motocicleta, usados como espacios sociales donde diferenciarse, sino también el segmento que delimita la marca HD. Las relaciones en este tipo de consumidores no se producen en base a una identificación con una imagen genérica del consumidor harlista. Muy al contrario, lo hacen en base a un particularismo altamente diferenciador que tiende a minimizar hasta abolirlo el valor del 'otro' en el consumo.

H: “(...) De hecho, este verano iremos estos 7 amigos, que somos 14, o sea 7 motos, 14 amigos, y nos vamos a los Alpes en Francia, y allí subirán 14 personas, también del mismo entorno (...). Y allí pues nos reuniremos y nos lo pasaremos bien (...). No, en común, mira, te lo diré bien claro. Que la mayoría somos matrimonios, casi todos... no, te diría, todos, excepto uno que es soltero. En común tenemos que somos unas personas de una edad al entorno de los 50 años o más. En común tenemos que todos tenemos una responsabilidad detrás, con lo cual queremos volver a casa, no vamos a hacer el indio por la carretera, vale. En común tenemos, de momento, toquemos madera, una estabilidad económica, o un trabajo de responsabilidad ... "(EO2).

\section{Comunitarismo particularista defensivo: relaciones en los Motoclubs (no exclusivos de HD)}

El último discurso referido a las relaciones lo encontramos en los consumidores de posición socioeconómica media-baja, miembros de los autodenominados Motoclubs (no formado exclusivamente por motoristas de HD) del GD3, las EA1 y EA4.

Las relaciones vuelven a aparecer como un elemento esencial de las prácticas de consumo, así como para la definición de la identidad colectiva de estos consumidores. Aquí la identidad primordial es la de "motero", no la de harlista; la marca que se consume es una cuestión secundaria, o por lo menos relativizada y no tan central como en los modelos anteriores. Coinciden con el lenguaje empleado por los miembros del HOG al verbalizar sus relaciones a través de la "camaradería" y la "solidaridad", así como también con la "fraternidad" o la "familia". Ello denota la polisemia de estas expresiones, según se usan en modelos distintos de relaciones (Maas, 2013). Estos valores no se limitan a los consumidores de HD como ocurre en el HOG, sino que se hacen extensivos a los moteros en general. Estos consumidores, sin embargo, los atribuyen 
fundamentalmente a las relaciones entre los miembros de sus respectivos Motoclubs.

Aquí las relaciones de consumo y de amistad se articulan sin una clara separación. Para estos consumidores la fortaleza de sus vínculos internos es un elemento diferencial respecto a otros tipos de grupo. Arguyen tener un compromiso fuerte con los otros miembros del grupo, significando estas relaciones con la confianza y una fuerte intensidad. Los MC's suelen ser grupos con un número de miembros relativamente reducido, lo que facilita el desarrollo de este tipo de relaciones. A diferencia de los otros modelos, aquí el grupo que forma el MC tiene una centralidad importante en la vida de los consumidores.

El discurso explícito expresa el carácter lúdico del grupo, aunque sin mostrar el carácter auxiliar e instrumental del 'otro'. Según dicen, se trata de pasarlo bien estando con el 'otro', en un tipo de socialidad cercana a la planteada por Simmel (1971). Pero el disfrute no lo es todo en este discurso, los consumidores ponen de relieve un cierto sacrificio con el conjunto del grupo. Cada miembro debe mostrar disposición a asumir un compromiso fuerte con los demás y el grupo en su conjunto. Así se llega incluso a la pérdida de individualidad en favor del grupo. El grupo adquiere relevancia por sí mismo, proporciona identidad a sus miembros y hasta un nombre, por el cual se le considera como entidad propia. Las acciones de los miembros del grupo (considerados individualmente) pasan a ser acciones del grupo, de modo que cada miembro es representante del grupo. Es importante, así, para estos consumidores, no cometer 'errores' o ser cautelosos en determinadas acciones, dado que lo que hace un miembro del grupo, lo hace como grupo (H: "había un C. -nombre grupo- haciendo el gilipollas...", GD3).

Las relaciones mantenidas entre grupos de moteros se encuentran generalmente reguladas por un principio de reciprocidad. Se entiende así que aquello que hace el grupo -sea positivo o negativo- a otros grupos les será devuelto (o ellos tendrán que devolver) en otra ocasión. Esto suele traducirse en una particular lógica del don (Mauss, 1924; McCracken, 1988), consistente en la asistencia pacífica y amistosa a eventos o actividades organizados por grupos ajenos. Las relaciones intergrupales, en este caso, particularmente entre MC's, se orientan a mostrar a los otros grupos la cortesía rendida, el respeto al grupo, que es lo que será devuelto (y esperado) en futuros eventos. Cabe añadir que existen otras lógicas, marcadas por la rivalidad entre MC's distintos, que no han sido exploradas en este trabajo. 
El aspecto relativo a las obligaciones, a las normas internas al grupo, ocupa un espacio importante en el discurso de los MC's. Por ejemplo, cobra cabal importancia en la discusión en el grupo el proceso de aceptación de un nuevo miembro. Este proceso requiere un reconocimiento del candidato por parte de los miembros de pleno derecho; lo que supone pasar por el ritual de unas "salidas" de prueba antes de proporcionar al nuevo miembro los signos identificativos del grupo (chaleco, parche, etc.) e indicarle su pertenencia al mismo. Los criterios para el acceso se centran en las acciones y actitudes del candidato, más que no en la marca de la motocicleta. El compromiso mostrado ante los demás miembros del grupo es lo que acaba determinando, según se expresa, la entrada en el grupo. De este modo no se conciben relaciones en el seno del MC de tipo efímero, cambiantes o débiles, donde el otro pueda ser prácticamente un desconocido o alguien con quien las hostilidades y los conflictos sean probables. Se trata de un sistema normativo que tiende a comportar, a la vez, mayores cuotas de coerción y cohesión que en los modelos de relación anteriores.

H: "En primer lugar, nosotros no valoramos la moto que llevas, valoramos como persona como te comportas, eso para empezar. Porque, además, eso ya me lo dijeron a mí una vez, porque no siempre he sido usuario de custom, he tenido de todo tipo de motos. $\mathrm{Y}$ en una ocasión que fuí a una concentración custom le dije: oye no tengo una custom, tengo una R, una R es una moto de carretera. Y me dijo: a mí me da igual lo que lleves debajo del culo. Yo ya te conozco a tí. Esto fue una frase que me quedé para mí también no. Y... aparte de eso, nosotros como te he dicho antes, somos un poco una familia, y aquí para entrar en C., primero debes frecuentar con nosotros durante un tiempo, sin ningún tipo de parche ni de chaleco ni de identificación ni de nada. Tienes que venir con nosotros, debes rodar con nosotros, tienes que salir el domingo...

H: Y convivir...

H: (...) Nuestro chaleco, nuestro parche, el de cualquier club, el de cualquier MC, el de cualquier peña motera, dile como quieras, implica que si yo hago el gilipollas estoy haciendo el gilipollas en nombre de club. (...) Vale, entonces esto implica involucrarte con las demás personas. Por eso nosotros antes de coger nadie, no es que se le haga una prueba, o que tenga un período de adaptación, sino que por su bien y por el nuestro, primero pruébalo, vienes, ruedas con nosotros, vienes a comer, vienes a, donde te sea, el que hacemos. Y después de un tiempo decidimos por ambas partes si adelante o no. "(GD3). 
Por otra parte, se hace patente el efecto de las posiciones sociales de los consumidores en la afinidad en los gustos y en determinadas prácticas de consumo. Éstas aparecen como proyecciones de los habitus compartidos que, efectivamente, producen espacios para el intercambio de relaciones (Bourdieu 1979: 240). Asimismo, juegan como material para la expresión identitaria y el reconocimiento social. El interés por la mecánica de las motocicletas, la asistencia a conciertos, fiestas y concentraciones moteras, así como salidas "gamberras", se relata como lo más significativo. Hallazgos similares con consumidores de Harley los apotran Bagozzi y Dholakia (2006).

Finalmente, más allá de los elementos socioculturales analizados, podemos interpretar el sistema de representaciones y normas que caracterizan este modelo de grupalidad desde la perspectiva del ideal del Yo (Freud, 1921). Las significaciones y vivencias asociadas al grupo no responderían al enaltecimiento del Yo, ni únicamente a la consecución de placeres inmediatos, como parecía ocurrir en los modelos anteriores, en especial el primero. Las relaciones en el grupo tienen como referentes proyectos y aspiraciones, así como modelos culturales y de conducta en los que no están ausentes las obligaciones y el esfuerzo de adecuación normativa, en este caso, al grupo. Esta cuestión es relevante teniendo en cuenta que se trata de un grupo creado por afinidad electiva.

Algunos de estos consumidores relatan que han tenido que abandonar el consumo de Harley por razones económicas. Ello los lleva a invertir la lucha (perdida) por el estatus simbólico en el campo de la HD, definiendo otra lógica simbólica y espacio de legitimidad. Eso es, usan una lógica basada en el tópico de la 'autenticidad motera' -Harley o no- para configurar el reconocimiento de su identidad grupal MC, en línea con los que señalan Grignon y Passeron (1992) y Elliot y Davies (2006) en sus trabajos. De este modo proyectan en el grupo un modelo ideal que seguir, al que adscribirse por su identidad motera 'auténtica'. Sobre ésta pivota la identificación recíproca entre consumidores, lógica motivacional de las relaciones en el sí del grupo. 


\section{Conclusiones e implicaciones: el 'vínculo social' fallido en el con- sumo de Harley-Davidson}

En el presente trabajo hemos analizado, mediante metodología cualitativa, el sentido que tienen los vínculos generados en torno al consumo de Harley-Davidson. De un modo más concreto, hemos abordado el contenido sustantivo de estos vínculos para sus consumidores.

El marco general del trabajo ha sido el debate acerca del papel que tienen los vínculos en determinadas formas de consumo actuales, especialmente planteado por la literatura referente a las comunidades marca. Asimismo, hemos incorporado algunas perspectivas de análisis que, tradicionalmente asociadas a la sociología del consumo, han sido poco aplicadas al fenómeno de los vínculos en el consumo de marcas. Estas aportaciones nos han permitido avanzar en una interpretación en términos de ambivalencia de los vínculos en el consumo, que se aleja de la celebración de formas de vínculo postmoderno.

El análisis interpretativo de los discursos nos ha mostrado cómo los vínculos desarrollados en el consumo de esta conocida motocicleta mantienen una estructura diversa, con dinámicas contradictorias más o menos asociadas a aspectos socioculturales, emocionales, así como a las posiciones sociales de los consumidores. Los procesos de diferenciación e identificación social se han revelado fundamentales para la generación y mantenimiento de los vínculos analizados.

Estos procesos, como hemos visto en los resultados, pueden interpretarse como la expresión de luchas simbólicas entre grupos sociales, desarrollados incluso dentro de un mismo segmento/marca de consumidores. Ello pone en evidencia el juego por el poder simbólico, así como por el reconocimiento y el cierre social que entraña el consumo de HD. Constatamos que los vínculos internos de los distintos grupos analizados, más o menos cercanos al modelo de comunidad posmoderna, adquieren un carácter fundamentalmente particularista, con unos universos simbólicos cerrados y autoreferidos.

En cuanto al 'vínculo social', no hemos observado la predominancia clara de ninguno de sus componentes. Sí han sido evidentes, en cambio, las exclusiones, tanto reales como simbólicas entre consumidores concebidos como 'insalvablemente' diferentes, así como el rechazo de toda traza de alteridad. Estos procesos son coherentes con la tendencia observada en los discursos hacia la homogeneización interna de los distintos 
grupos que componen el conjunto de los consumidores harlistas. Según lo analizado, ésta puede darse por la vía de la salida o expulsión del grupo, cuando la diferencia es demasiado grande con el universo simbólico del grupo, o cuando se pretenden ciertos privilegios individuales (HOG); por otro lado, por la vía de la reducción de las diferencias individuales entre los miembros del grupo, antes de la entrada en el mismo, en el caso de los MC's.

En los discursos, particularmente entre los consumidores de posición media y media-alta, se observa un modelo de construcción objetiva de las relaciones con los demás. La realización subjetiva del 'otro', es decir, la atención puesta sobre aquello que el 'otro' recibe y a cómo lo recibe en los vínculos, es una dimensión prácticamente inexistente en el relato de los consumidores.

Por otra parte, hemos observado que algunos discursos revelan el compromiso fuerte que adquieren los vínculos (de las relaciones, fundamentalmente) entre consumidores, como ocurre con los MC's. Sin embargo, se trata de un compromiso normativizado que coexiste con la pérdida de autonomía personal a favor del grupo. Los vínculos no dejan de presentarse con un carácter cerrado y con un modelo de socialidad privada sin ningún proyecto integrador.

El consumo de marcas, por tanto, y en particular el de Harley-Davidson, difícilmente puede representar un contexto de aceptación mutua entre consumidores. Y por ello, un modelo de vínculo basado en el reconocimiento de los 'otros' en tanto que 'otros' (Barbeta, 2011). Los vínculos en el consumo de HD no se caracterizan por su dimensión social, sino por la creación de comunidades de afines. Afinidades en el consumo en base a formas de identidad, deseos, símbolos y códigos comunes, así como la creación de círculos más bien cerrados. La necesidad de aprobación y el reconocimiento lúdico y narcisista son también elementos centrales para lo cual se tienen en cuenta a los demás en el consumo.

Es bien posible, de este modo, que desmantelados los mecanismos institucionalizados de la era keynesiana-fordista, y en un contexto de remercantilización generalizada de la vida, el consumo privado se muestre como uno de los ámbitos de reconstrucción de las identidades y socialidades que en los últimos años se han visto en crisis (Alonso y FernándezRodríguez, 2013; Castel, 2003). Sin embargo, con este trabajo pretendemos afirmar que se trata de una salida fallida de la reconstrucción de la solidaridad y el 'vínculo social' que encuentra en la universalidad y la aceptación de la alteridad la base de su desarrollo. 


\section{Bibliografía}

Aaker, d. (1991). Managing Brand Equity: Capitalizing on the Value of a Brand Name. New York : Free Press.

Alonso, L. E. (1998). La mirada cualitativa en sociología. Madrid: Fundamentos.

Alonso L. E. y Fernández Rodríguez, C. J. (2013). Los discursos del presente. Un análisis de los imaginarios sociales contemporáneos. Madrid: Siglo XXI.

Arnould, E. J. y Thompson, C. J. (2005). "Consumer Culture Theory (CCT): Twenty Years of Research". 31,868-82.

Askegaard, S. y Jeppe, T. L. (2011). "Towards an epistemology of consumer culture theory Phenomenology and the context of context". Marketing Theory, $11,4,381-404$.

Bagozzi, R. P., y Dholakia, U. M. (2006). "Antecedents and purchase consequences of customer participation in small group brand communities". International Journal of Research in Marketing, 23, 45-61. doi:10.1016/j.ijresmar.2006.01.005

Barbeta Viñas, M. (2018). "Los «otros» en el consumo de Harley-Davidson: análisis de los lazos exogrupales en las «comunidades marca» ". Pensar la Publicidad: Revista Internacional de Investigaciones Publicitarias, 12: 33-51.

Barbeta Viñas, M. (2014). "De los vínculos en el consumo al consumo en los vínculos: análisis de las formas de sociabilidad en las prácticas de consumo ecológico", Revista Española de Sociología, 22: 67-95.

Barbeta Viñas, M. (2011) ."Lo que marcan las marcas: una aproximación sociohistórica al consumo de marcas". Política y Sociedad, 48 (1): 95-116.

Bauman, Z. (2001a). Community: Seeking Safety in an Insecure World. Cambridge: Polity.

Bauman, Z. (2001b). The Individualized Society. Cambridge: Polity Press.

Bauman, Z. (2007). Consuming Life. Cambridge: Polity Press.

Bardhi, F.; Eckhardt, G. M. y Arnould, E. (2012). "Liquid Relationship to Possessions". Journal of Consumer Research, 39, 3, 510-29.

Belk, R. y Tumbat, G. (2005). "The cult of Macintosh", Consumption, Markets, and Culture, 8, 3, 205-17.

Blocker, C.; Houston, M.; Flint, D. (2012). "Unpacking What a 'Relationship' Means to Commercial Buyers: How the Relationship Metaphor Creates Tension and Obscures Experience". Journal of Consumer Research, 38, 5, 886905.

Boltanski, J. L. (1990). Love and Justice as Competences. Cambridge: Polity Press.

Borras, V. (1998). El consumo, un análisis sociológico: la estructuración del consumo y los grupos sociales en la Región Metropolitana de Barcelona. Barcelona: Cedegs.

Bourdieu, P. (1979/1984). Distinction: a Social Critique of the Judgement of Taste. London: Routledge.

Bourdieu, P. (1980). Le Sens pratique. París: Minuit.

Bowlby, J. (1979). Vínculos afectivos: Formación, desarrollo y pérdida. Madrid: 
Morata.

Buss, D. y Chiodo, L. M. (1991). "Narcissistic acts in everyday life". Journal of. Personality, 59: 179-215.

Carlson, B. D., Suter, T. A. y Brown, T. J. (2008). "Social versus psychological brand community: The role of psychological sense of brand community". Journal of Business Research, 61, 284-91.

Castel, R. (2003). L'insecurité sociale. París: Seuil.

Chaney, D. (1996). Lifestyles. London : Routledge.

Cisek, S.; Sedikides, C.; Hart, C. M; Godwin, H.; Benson ,Va y Liversedge, S. (2014). "Narcissism and consumer behaviour: a review and preliminary findings". Frontiers in Psychology, 5:232.

Conde, F. (2008). "Los grupos triangulares como espacios transicionales para la producción discursiva: un estudio sobre la vivienda en Huelva". En Gordo A. y Serrano, A. Estrategias y prácticas cualitativas de investigación social, Madrid: Pearson Educación. 155-188.

Conde, F. (2009). Análisis sociológico del sistema de discursos. Madrid: Centro de Investigaciones Sociológicas.

Cova, B. (1997). "Community and Consumption: Towards a Definition of the 'Linking Value' of Productor Services". European Journal of Marketing, 31, 34,297-316.

Cova, B. y Cova, V. (2002). "Tribal marketing: the tribalisation of society and its impact on the conduct of marketing". European Journal of Marketing, 36, 5/6, 595-620.

Cova, B.; Kozinets; R. V. y Shankar, A. (2007). Consumer tribes. Oxford and Burlington: Butterworth-Heinemann.

Douglas, M. y Isherwood, B. (1979). The World of Goods, New York: Basic.

Elliott, R. y Davies, A. (2006). "Symbolic Brands and Authenticity of Identity Performance". En: Schroeder, J. and Salzerg-Morling, M. (eds.), Brands Culture. London: Routledge. 155-169

Ewing, M.; Wagstaff, P. y Powell, I. (2013): «Brand rivalry and community conflict», Journal of Business Research, 61, 4-12.

Fournier, S. (1988). "Consumers and their Brands: Developing Relationship Theory in Consumer Research". Journal of Consumer Research, 24,1, 343-373

Fournier, S., Sensiper, S., McAlexander J. H. y Schouten J.W. (2000). "Building brand community on the Harley-Davidson posse ride". Harvard Business School Case, Reprint N. 501009, Milwaukee.

Freud, S. (1914/2004). On the Introduction of Narcisism. New York, Penguin Books.

Freud, S. (1921/2004). Mass Pshicology and Analysis of the I. New York: Penguin Books.

Gebauer, J.; Füller, J. y Pezzei, R. (2012). "The dark and the bright side of cocreation: Triggers of member behavior in online innovation communities". Journal of Business Research, 66, 9, 1516-1527. 
Grignon, C. y Passeron, J. C. (1992). Lo culto y lo popular. Buenos Aires: Nueva Visión.

Habermas, J. (1980). The Theory of Communicative Action (2 vols). Boston: Bacon Press.

Hikman, T. y Ward, J. (2007). "The Dark Side of Brand Community: Inter-Group, Stereotyping, Trash Talk and Schadenfreude". En: Fitzsimons, G. y Morwitz, V., Duluth. Advances in Consumer Research, 34, MN: Association for Consumer Research, 314-319.

Holt, D. B. (1994). "How Consumers Consume: A Typology of Consumption Practices". Journal of Consumer Research, 22:1-16.

Husemann, K.C.; Ladstaetter, F. y Luedicke, M. K. (2015). "Conflict Culture and

Conflict Management in Consumption Communities". Psychology and Marketing, 32, 3, 265-284

Izquierdo, M. J. (1998). El malestar en la desigualdad. Madrid: Cátedra.

Izquierdo, M.J. (2003). "El cuidado de los individuos y de los grupos: quién se cuida. Organización social y género", Intercambios, Papeles de psicoanálisis, $10,70-82$.

Laplanche, J. y Pontalis, J.-B. (1967/1996). Diccionario de Psicoanálisis. Barcelona: Paidós.

Lasch, C. (1979). The Culture of Narcisism. New York: Norton.

Lee, M. J. (1993). Consumer Culture Reborn: the Culture Politics of Consumption. London: Routledge.

Lipovetsky, G. (1983). L'ère du vide. Essais sur l'individualisme contemporain. Paris: Gallimard.

Lipovetsky, G.(2006). Le bonheur paradoxal. Essai sur la société l'hyperconsommation. Paris : Gallimard.

Maas, K. M., (2013). "Making Sense Of Motorcycle Brotherhood: Women, Branding, And Construction Of Self”. AllTheses, Dissertations, and Other Capstone Projects. Paper 238.

Maffesoli, M. (1988). Times of Tribes: The Decline of Individualism in Mass Society. Thousands Oaks CA: Sage.

Maffesoli, M. (1996). Contemplation Of The World : Figures of Community Style. Minnessota: University of Minnessota Press.

Martin, D.; Schouten, J.; Mc Alexander, J. H. (2006). "Claiming the throttle: multiple femininities in a hyper-masculine subculture", Consumption, Markets and Culture. 9, 3,171-205.

Marx, K. (1844/2007). Economic and Philosophic Manuscripts of 1844. New York: Dover Publications.

Marzocchi, G.; Morandin, G.; Bergami, M. (2013). "Brand communities: loyal to the community or the brand?" European Journal of Marketing, 47, Iss: $1 / 2,93$ 114.

Mauss, M. (1923/1990). The Gift: Forms of Function of Exchange in Archaic Societies, New York: Routledge. 
Maturana, H. (1988). "Reality: the search for objectivity or the quest for a compelling argument". The Irish Journal of Psychology, 9, 1, 25-82.

McAlexander, JH. (2011): «Communitas Interruptus: The Limits of Loyalty», Proceedings of the European Association for Consumer Research. 19, 401-405.

Mc Alexander J. H., Schouten, J. W. y Koening, Harold F. (2002). "Building brand community", Journal of Marketing. 66, 38-55.

Mc Alexander, J. H.; Kim, S.K. y Roberts, S. D. (2003)."Loyalty: The influence of satisfaction and brand community integration", Journal of Marketing Theory and practice. $11,4,1-11$.

Mc Cracken, G. (1988). Culture and Consumption. Bloomington: Indiana University Press.

Merton, R K. y Barber, E. (1976). "Sociological ambivalence”. En: Merton, R. K. Sociological ambivalence and others essays. New York: The Free Press. 3-31.

Miller, D. (1987) Material Culture and Mass Consumption. Cambridge: Basil Blackwell.

Miller, D. (1999). A Theory of Shopping. New York: Cornell University Press.

Minsky, R. (1998). Psychoanalysis and Culture: Contemporary States of Mind. UK: Rudgers University Press.

Muñiz, A. M. y O'Guinn, T. (1996). "Brand Community and the Sociology of Brands". En: Special Sessions "Comunities of Consumption: A Central Metaphor for Diverse Research". Kim P. Corfman and John G. Lynch, eds. Advances in Consumer Research, 23, Provo, UT: Association for Consumer Research. 265-66.

Muñiz, A. M. y O'Guinn, T. (2001). "Brand community and the sociology of brands", Journal of Consumer Research, 27, 412-32.

Muniz, A. M. y Hamer, L. O. (2001). "Us Versus Them: Oppositional Brand Loyalty and the Cola Wars”. En: Gilly, M y Meyers-Levy, J. Valdosta, (eds.), Advances in Consumer Research, 28, GA: Association for Consumer Research. 355-61.

Ollivier, M.; Gauthier, G. y Truong, A. (2009). "Cultural classifications and social divisions: A symmetrical Approach”, Poetics 37, 5-6, 456-473.

Patterson, M. y O'Malley, L. 2006. "Brands, consumers and relationships:A review". Irish Marketing Review. 18,1 \& 2,10-20.

Santos, E. D. S. M., Silva, D. D., Braga Junior, S. S., y Do Nascimento, C. A. X. (2017). "Comportamento do consumidor da Comunidade de Marca HarleyDavidson e a influência do self-expandido". Revista Brasileira de Marketing, 16(1), 98-114.

Schembri, S. (2009). "Reframing brand experience: The experiential meaning of Harley-Davidson". Journal of Business Research, 62:1299-310.

Schouten, J. W.; Martin, D.; McAlexander, J. (2007). "The evolution of a subculture of consumption". En: Cova B.; Kozinets; R. V. y Shankar, A. Consumer tribes. Oxford and Burlington:Butterworth-Heinemann. 67-75. 
Schouten, J.W. y Mc Alexander J. H. (1995). "Subcultures of consumption: an ethnography of the new bikers", Journal of Consumer Research, 22:43-61.

Sedikides, C., Cisek, S. y Hart, C. 2007. "The I That Buys:Narcissists as Consumers", Journal of Consumer Psychology. 17 ,4,254-57.

Sennett, R. (1979). The Fall of Public Man. New York: Penguin Books.

Sennett, R. (2012). Together: The Rituals, Pleasures and Politics of Cooperation. New

York:Penguin Books.

Severiano, M. F. (2005). Narcisismo y Publicidad. Un análisis psicosocial de los ideales del consumo en la contemporaneidad. Buenos Aires: Siglo XXI.

Simmel, G. (1971). On Individuality and Social Forms: essays of Georg Simmel. Chicago: University of Chicago Press.

Simmel, G.(1911/1988). Sobre la aventura. Ensayos filosóficos. Barcelona: Península.

Sherry, J. (1983). "Gift Giving in Anthropological Perspectives". Journal of Consumer Research. 10,2,157-168.

Veblen, T. (1899/1994). The theory of the leisure class. New York: Mcmillan.

Wallace, H. y Baumeister R. F. (2002). "The performance of narcissists rises and falls with perceived opportunity for glory". Journal Personality and Social Psychology. 8, 819-834.

Weber, M. (1922/1978). Economy and Society: an Outline of Interpretative Sociology. Berkley and Los Angeles: Calofornia University Press.

Yates, B. (2000). Outlaw Machine: Harley-Davidson and the Search for the American Soul. New York: Brodway Books.

\section{Nota biográfica:}

Marc Barbeta Viñas es doctor en sociología por la Universidad Autónoma de Barcelona. Trabaja como profesor asociado por la Universidad Autónoma de Barcelona y la Universidad de Gerona. Ha sido investigador en el departamento de sociología de la UAB en grupos de investigación como el GESES e Iphigenia. Sus principales líneas de investigación son: la sociología del consumo y la cultura, así como los métodos y técnicas de investigación cualitativa. También ha trabajado en investigaciones en el ámbito de la familia, el género, la educación y la desigualdad social.

Dirección institucional: Departament Sociologia, Universitat Autònoma de Barcelona. Avinguda Eix Central. Edifici B, 08193 Bellaterra (Cerdanyola del Vallès)

Correo electrónico: marc.barbeta@gmail.com

Orcid: 0000-0002-3630-3367 
Recibido: 07-05-2018

Aceptado: 10-04-2019

(c) Licencia Creative Commons Reconocimiento (CC BY 4.0)

OBETS. Revista de Ciencias Sociales, Vol. 14, nº 1, 2019; pp. 17-48. DOI: 10.14198/OBETS2019.14.1.02 